\title{
The Architecture Design of Dynamic Information Acquisition Technology of Crop Yields
}

\author{
Yiqing Ye, Zhepeng Wei, Wangbo Zheng, Xiaoying Huang* \\ Tianmu College of Zhejiang A\&F University, Lin'an 311300, Zhejiang, China \\ ahxyhn@yahoo.com.cn
}

\begin{abstract}
Lack of access to technical information for existing agricultural production and low efficiency, for example Chinese cabbage, , proposes a high efficiency, the precision dynamic cloud acquisition theory and methods, while the use of the Hadoop framework taken MapReduce, Combined with Skyline queries technology, Build a cloud-based agricultural production and marketing information sharing platform, Agricultural production information in real time dynamic acquisition, processing and transmission in a timely manner, agricultural production information analysis, storage calculation result, agricultural production and price forecast information is valid, the timely release, Improve the effectiveness of the data quality of agricultural products and information services, Thus solve the problem to control price volatility of agricultural products, agricultural information collection to release lag, So that scientific and effective decision-making, and the macro-management of the balance between supply and demand of agricultural products.
\end{abstract}

Keywords: agricultural, Yield Information, Dynamic acquisition

\section{Introduction}

Agricultural price stabilization and security is the foundation of the smooth running of the entire national economy and social, harmony and stability. Price fluctuations in agricultural products both at home and abroad in recent years, especially in the domestic prices of agricultural products like a "roller coaster", "herd, a coax under the" volatility alternately, causing serious waste of limited agricultural resource allocation brought

huge losses to the agricultural development and farmers, and a serious impact on people's daily lives and social stability; highlights China as the most populous country and the large agricultural country, the grim reality of the macro-management of agricultural products.

Domestic and international studies have shown that under the market mechanism, the supply and demand of agricultural products important factor is price volatility. Rule in accordance with the "cobweb theory" as well as "on-demand production quotas", the only producer to grasp market prices, trends in consumer demand and production structure prediction, prediction information, and accordingly the production structure and scale adjustment, be possible to achieve agricultural products supply and demand balance. "Ups and downs" conducive to the elimination of agricultural prices, help to reduce the adverse effects caused by the agricultural production in the cobweb phenomenon. The Zhu Wanhong (2010)[1] pointed out that the sharp fluctuations in vegetable prices is caused by the lack of information., Ma Guoying (2011)[2] study asymmetric information to the speculation opportunities, leading to poor sales of vegetable origin, pin your premium. Thus establishing a comprehensive, fair, accurate, timely, transparent and effective agricultural information system, so that dynamic information collection, processing, transmission and timely information supply system, the scientific decision-making and an important prerequisite for effective control of the volatility of agricultural prices and key.

\section{The Significance of Information Acquisition of Agricultural Outputs}

Agricultural information is an essential resource for agricultural production activities, timely and accurate grasp and use of agricultural information, agricultural production and business activities of the structural adjustment and macroeconomic management decision-making basis.

Basic supply of agricultural products in China from three aspects: imports this year, on the year-end inventory levels and this year production, due to the limited total capacity by warehouse inventories of agricultural products, small agricultural imports, so this year production proportion play a decisive role, thus the collection of information critical to agricultural production, but also due to the long cycle of agricultural production, the market, many links involved in a wide range, and the supply and demand relationship is dynamic, so that the data is accurate, real-time acquisition there are difficulties, coupled with China agricultural information system is not perfect, agricultural production, circulation and consumption of various aspects of severe asymmetry, especially the majority of producers rely on rumors of neighbors and local TV information, lack of information on the supply channels, regional limitations of information very large.

Therefore, the establishment of a comprehensive, fair, accurate, transparent information system of agricultural products, timely and comprehensive dynamic acquisition, processing, and transport agricultural production information is the premise of the agricultural production and forecast information service.

Fund Project : 1 .The Humanity and social science planning Foundation of Ministry of Education of China(No.12YJA870008)

2.Undergraduate Science and Technology Innovation Items of Tianmu College of Zhejiang A\&F University (No.TMKC1239)

* Corresponding Author: Professor, Zhejiang A\&F University. Email:ahxyhn@yahoo.com.cn 


\section{The Methods and Brief Introduction of Agricultural Information Acquisition}

By domestic researchers on the study of agricultural information collection methods, is not difficult to find, a major agricultural information websites, can query to the market price of many agricultural products, market dynamics, as well as the prices of agricultural products warning prompt. But found a lot of information synchronization speed is slow, and the information lag, resulting in not timely guidance on the structure of agricultural production. Mainly because the price of agricultural products information acquisition mode artificial sources of information collected from the major farmers market, and then a lot of form data through the keyboard, mouse manually entered into the computer for processing, to be published. Its heavy workload, error rate, data rendering speed is slow. And there may be some human factors. Looking at our existing agricultural information services and studies show that the reality of the status quo, traditional agricultural information collection and the way it releases, backward, and the heavy workload. Create intelligent agricultural information collection is an urgent need for future agricultural development and trends. The project, Chinese cabbage, for example, the use of cloud computing makes intelligent and fast acquisition and analysis of agricultural information.

\section{Methodology of Information Acquisition}

Release lag and other issues to improve the traditional agricultural information collection, access to real-time, accurate, and efficient agricultural production information, propose an information intelligent direction, high efficiency, precise dynamic cloud acquisition theory and methods, the use of computer technology to obtain information of Chinese cabbage, to avoid the evils of artificial collection

Cabbage regional production forecast is mainly based on the area of Chinese cabbage seed sowing rate of sowing date, seed germination rate, survival rate of late, and the growth of state predict. Dynamic monitoring the production of agricultural products of the time period, can be divided into three periods of cabbage First day cabbage growth data upload and analysis of the results obtained, then a week, month, quarter units cabbage growth data were collected and analyzed results obtained, the render icons manner.

\subsection{Sample Experiment of Selected Farmlands}

Traditional the farmland environment system data sampling point layout method main diagonal cloth points, plum-shaped cloth point method, checkerboard cloth point method, serpentine (S-shaped) distribution law. In the experiments, due to the acquisition of the main targets for farmland environment, select the target plots smaller but farmland soil, cabbage cultivation is relatively evenly distributed, integrated from savings sampling the workload considering choose the quincunx cloth point method for deepening and improvements. Select one hectare square farmland as an experimental site (to ensure the accuracy of the data, the sampling points should steer clear position in the gutter and the edge of the compost pile) and reasonably uniform planting experiment farmland (the same as the number per square meter planting cabbage), the first step in every four square meters of farmland (labeled $\mathrm{A} 1, \mathrm{~A} 2, \mathrm{~A} 3, \ldots)$ in randomly selected as experimental samples and mark a cabbage, the second step, to every eight square meters of farmland (B1, B2, B3 ...) as a unit and then randomly selected a cabbage as a test sample and mark the third step, each 16 square meters of farmland $(\mathrm{C} 1, \mathrm{C} 2, \mathrm{C} 3$...) as a unit and then randomly selected strains cabbage as experimental samples and mark (sample shall be repeated), statistical one hectare of farmland samples for the $Z, Z=\Sigma A$ $+\Sigma \mathrm{B}+\Sigma \mathrm{C}=4375$ growth situation information for each sample to be collected, and to estimate the yield, timely and rapid transmission, and hectares growth status and yield more accurate estimates.

\subsection{Analysis and Information Acquisition of Cabbage Seeding Rate}

Seed seeding rates could start to investigate the cabbage seed market from the farmers market, market survey collection or select a place from around the seed market sales, uploaded to a computer for storage and analysis, preliminary calculation of the large cabbage seed sowing rate.

\subsection{Analysis and Information Acquisition of Cabbage Germination Rate}

Affect the cabbage germination are many external factors, such as the fertile and heavy metal content of the soil condition, weather conditions, water demand conditions, the experimental data on the sample analysis, Out the seed germination rate can be calculated from the experimental fields, and can seed germination growth condition analysis, and timely seed replacement, in order to ensure the yield of agricultural products.

\subsection{Later Stage of Cabbage Survival Rate}

The survival rate of cabbage directly affect the entire production of cabbage, which not only cabbage death statistics and remove also its death situation analysis, especially indepth study of pests and diseases, so as not to affect other plantsgrowth. The project is mainly based on the chemical instrument acquisition the cabbage growth process in the various factors indicators, its intelligent analysis, to arrive yield results.

\section{Information Acquisition Technology of External Factors Which Affected Cabbage Production}

Meteorological factors, soil factors, pest factors cabbage can use now has the technology to detect.

\subsection{Modeling of Corresponding Meteorological Data}

Agricultural production has a close relationship with farmland meteorological factors, mainly the atmosphere, temperature, light, moisture. Build relationships will be able to get a good predictor of the effect of meteorological factors and yields. Agrometeorological yield prediction model[3] is: $y=y t$ yw. Where, y for the actual output, yt is the trend yield, yw meteorological yield. Previous use this formula to predict 
domestic[4-6] , various foreign[7] crop yields reached a very high accuracy. Therefore, you can predict the actual output of the cabbage.

\section{2. est Data Compilation of Heavy Metals in the Soil}

The soil data is one of the main object of study of farmland information, heavy metals affect food safety, the main heavy metal detection technology, the technique is mainly used in atomic absorption spectrometry method (AAS), UV-visible spectrophotometry (UV), atomic fluorescence spectrometry (AFS), electrochemical method[8]. Separately calculated error rate land with these four test samples (randomly selected sample of 100 land), four sample data averaging, data integration and transmission.

\subsection{Quick Information Acquisition of Cabbage Diseases and Insect Pests}

First in early prevention and treatment against diseases and insect pests on cabbage seeds, farmland the disinfected and go harm, rational use of fertilizers.

Good protection measures may still not fundamentally harm, so early detection and to determine the occurrence of crop pests and diseases, to improve the targeted spraying decision prevention and accuracy, to do the right remedy, demand spraying, and effectively reduce pesticide bring environmental pollution and medicines waste. The most common pest cabbage cabbage aphid, traditional information acquisition method of crop pests and diseases are usually the aid of a microscope or the naked eye to directly determine the type and extent of the pest, there are subjective workload, narrow coverage, low efficiency, cost higher shortcomings, and does not apply to precision agriculture need rapid access of information on crop pests and diseases, so that the image processing technology, spectrum technology, and multispectral and hyper-spectral imaging technology the cabbage cabbage aphid growth of rapid detection.

\section{Technology of Quick Access to Internal Factors Which Affected Cabbages}

The nutrition element is one of the main factors affecting crop growth and yield. Therefore, the the crop nutritional information to predict the yield of one of the important data quickly obtain.

\subsection{Quick Access to Crop Nutrition}

\subsubsection{Quick Access to Chlorophyll and Nitrogen During the Growth of Cabbages}

Nitrogen is one of the main factors affect the growth and yield of Chinese cabbage, Chinese cabbage leaf nitrogen content data acquisition, due to the similar trend between leaf nitrogen content and chlorophyll content through the determination of cabbage chlorophy content monitoring nitrogen nutrition. Using existing technology Japanese the MINOLTA company production of chlorophyll meter on the chlorophyll content of the information collection, Jia Liangliang ${ }^{[9]}$ found between jointing and flowering period chlorophyll meter readings and total nitrogen concentration of winter wheat canopy there is a significant correlation proposed by chlorophyll meter readings evaluation of crop nitrogen nutrition, which can be used in the analysis of the cabbage field in the block, the nitrogen content of the results can only do rough estimates, to rely on the nitrogen content of chlorophyll meter monitoring the whole field cabbage unrealistic rapid non-destructive testing methods including image and spectral analysis technology are working together quick access to the nitrogen can be applied to the growth process of Chinese cabbage.

He Yong[10], chlorophyll meter and spectrum analysis techniques to test the crop growth status characteristic curve and scatter diagram.
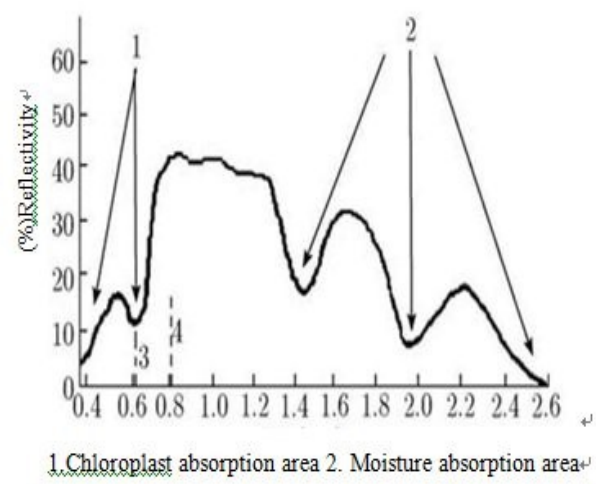

3. Red characteristic wavelength 4. Characteristic wavelengths of infrared light

Figure 1 Leaf spectral reflectance characteristic curve

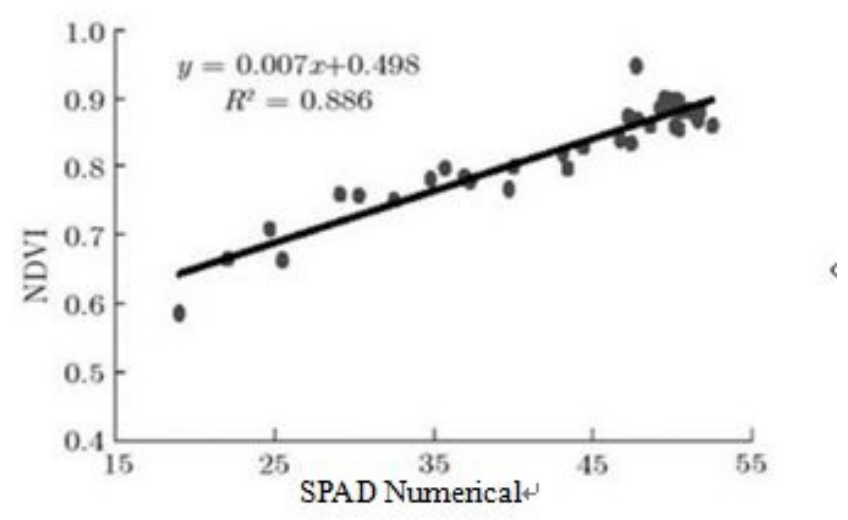

Figure 2 Scatter diagram between the measured values of the vegetation index and chlorophyll instrument

Non-destructive use of machine vision, speed, high efficiency, large amount of information, and so on, we can observe the leaves green component $\mathrm{G}$ and the chromaticity $\mathrm{H}$ component nitrogen content linear, and then combined with leaf green meter, can make to data becomes accurate and effective.

\subsubsection{Quick Access to Phosphorus and Potassium During the Growth of Cabbages}

In cabbage, phosphorus and potassium content of its production will have some impact, and thus take advantage of 
the existing spectral analysis technology collection, $\mathrm{Ke}[11]$ found that potassium canopy spectral characteristics and nitrogen similar effects. Zhao Chunjiang spectrum detection technology on the supply of tomatoes nutritional elements are reviewed that tomatoes nutritional elements can create supply model using Monte Carlo simulation method and line dynamic diffuse reflectance spectroscopy detection technology, which can make use of spectrum detection technology of Chinese cabbage establish nutrient the elements supply model for the intrinsic relationship between the analysis of nutritional elements and near-infrared spectroscopy.

\section{7. transmission of RFID to the Information of Crops}

Do information intelligent acquisition and rapid response analysis and, ultimately, the acquisition of information transfer, the acquisition of the rapid transmission of information can make the the cabbage growth status information to the analysis of stored released achieve higher synchronization rate, and thus the introduction of modern radio frequency technology to its implementation.

\subsection{The Conception and Application of RFID}

Typically, radio frequency identification is mainly composed of two parts, electronic tags and readers. Radio frequency identification is a non-contact automatic identification technology, automatic target recognition and access to relevant data through the alternating magnetic field or electromagnetic wave coupling. The RF transmission method has the following advantages: easy to use, fast data exchange speed, ease of maintenance and long service life, no physical contact can be completed to identify features that can be used to achieve the identification of multiple targets and moving targets.

Currently, RFID[12] technology has been widely used in the field of Computer Management, and penetrate into the post and telecommunications, materials management, security check, the standard card management and project various sectors of the national economy and people's daily lives. But rarely applied to agriculture, so the expansion of this technology which passed in cabbage growth factors

\subsection{The Application of RFID During the Growth of Cabbages}

Growth status and yield of cabbage chlorophyll meter, spectrum technology, machine vision technology, multispectral and hyper-spectral imaging technology to detect and analysis, and data transfer in data integration within the electronic tags, according to the operating frequency the antenna is transmitted to the reader, the antenna can also receive the RF signal from the transceiver, and authentication on the electronic tag identification information to be decoded, and other relevant information in the authentication identification information associated with the electronic label is transmitted to the host for processing. Chinese cabbage growth of information stored in the database.

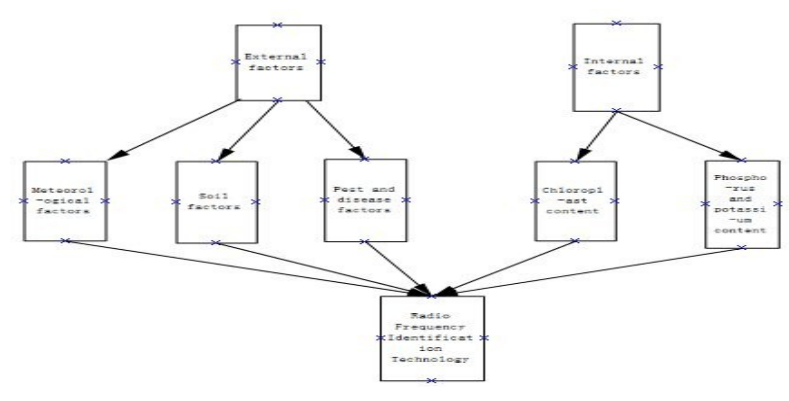

Figure 3 information collection framework

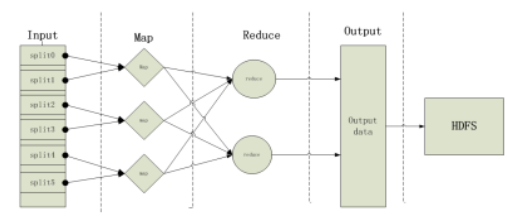

Figure 5 MapRuduce process flow

\section{Publishment of Growth Data of Cabbages}

The use of Hadoop framework MapReduce mode arithmetic results generated pattern with curve model specific pattern curve model analysis, and can be automated, digitized growth situation and the last cabbage production forecast. Forecast information released after processing, to achieve a fully automated information collection system.

\section{Conclusion}

The purpose of information sharing platform based on the production and marketing of agricultural products, Chinese cabbage as an example, its growth status and yield to do correlation analysis, and production-related information collected to do some research and discussion, cabbage ecological datacloud acquisition experiment equipment and related technology, and the pilot of the massive data processing using Hadoop Map / Reduce framework Skyline queries to data, so that producers can quickly and accurately search, filtering, statistical analysis of real-time agricultural productsdynamic information.

\section{References}

[1] Zhu Wanhong,countermeasures stable vegetables at reasonable prices in a new mode of production ,Chinese commerce 2010-12-01

[2] Ma Guoying, Yang Lei, Wang Jingjing, Chen Yongfu cause analysis of changes in the prices of vegetables and explore countermeasures 2011$07-15$

[3] Fang Jinglong,Meng QingYan,Wu Bingfang, Zhou Xintie, based model of agro-meteorological crop yield forecasting system, China Agricultural Meteorology, 2003 (5)

[4] Jin Yanxin, Zhang Li, Chen Hua, Jiangsu Province wheat production system analysis [J]. Agricultural Meteorology, 198910 (4)

[5] Zouyong Lin,Tang Zhicheng,Taobing Yan, agro-meteorological crop yield statistical forecasting model study [J]. China Agricultural Meteorology .1992,13(5)

[6] Wu Zhonglin, Chen Tieru,Li Linyi. Agrometeorological Yield forecasting methods to explore and Changchun, rice and corn production test reported [J]. Jilin Agricultural University .1993,15 (4)

[7] Yang XingWei,Zhou Hongmei,Li Jun. main producing countries of the world rice yield estimation feasibility study $[\mathrm{J}]$. The Applied 
Meteorology .1998,9 (2)

[8] Wang Shuyan,Tan Zunshe, Zhang Wei,Luan Fang ,Talking about agricultural products in heavy metal detection technology development, Luoyang City, the safety of agricultural products testing center 20094

[9] Jialiang Liang, Chen Xinping. Chlorophyll meter and plant nitrate concentration test comparison of the diagnostic accuracy of winter wheat in North China Agricultural University, 2007,22

[10] He Yong,Zhao Chunjiang, Wu Di, the Niepeng Cheng, Feng Lei. Crops - Environmental technology and sensors, Science in China: Information
Science .2010,40 (Suppl) :1-20

[11] Wang Keng,Shen Zhangquan, Abou the I potassium nutrition level of the rice canopy and leaf spectral characteristics of the Preliminary Report. Bulletin of Science and Technology. 1997,13

[12] He Quanjiang, Xia Lin.overview of the application of radio frequency identification technology. Modern building electrical .2011 8

[13] Linlin, Xin Junchang, Wang Guoren, Huangshan. Massive data based on the Map-Reduce efficient Skyline query processing computer of .2011 10 